\title{
Contribuição do Bitcoin na melhora da eficiência de um portfólio de investimentos
}

\author{
Bitcoin's contribution to improving the efficiency of an investment portfolio
}

Ana Luísa Moutinho ${ }^{1}$ e Roberto Silva da Penha ${ }^{2}$

\footnotetext{
${ }^{1}$ Universidade Federal de Minas Gerais, Brasil, Graduanda em Administração, e-mail: analuisamoutinho@hotmail.com ${ }^{2}$ Universidade Federal de Minas Gerais, Brasil, Doutorando Ciências Contábeis, e-mail: robertorrcontabilidade@hotmail.com
}

\section{Resumo}

Com o avanço das criptomoedas em especial o Bitcoin em relação população e das valorizações da mesma principalmente em 2017. Torna-se comum a comparação do Bitcoin com outros ativos, sendo utilizado para diminuir os riscos de uma carteira de investimentos, também passando a ser considerada uma nova classe de ativos. Diante deste cenário, levanta-se a seguinte questão de pesquisa: $O$ uso do Bitcoin pode trazer benefícios de otimização de uma carteira de investimentos? Caso sim, qual é a sua participação? A pesquisa possui abordagem quantitativa. $\mathrm{O}$ universo da pesquisa compreende 48 observações mensais, no período entre janeiro de 2014 a dezembro de 2017, para cada uma das variáveis utilizadas no estudo, a saber: (a) Ibovespa; (b) IBrX 50; (c) LFT; (d) ouro; (e) real-dólar; e (f) Bitcoin. O procedimento de cálculo para otimização da carteira se deu mediante o uso da ferramenta Solver do Microsoft Excel ${ }^{\circledR}$. Foi montada uma carteira com e sem Bitcoin, para responder ao problema de pesquisa. Os resultados apontaram que que os retornos dos portfólios são maiores em carteiras contendo o Bitcoin para os anos de 2015, 2016 e 2017, enquanto que em 2014 não se teve diferenças, exatamente o ano em que os retornos do Bitcoin foram negativos. Assim, o acréscimo do Bitcoin em um portfólio de investimento se mostrou vantajosa, permitindo trazer uma melhor otimização da carteira.

Palavras-chave: Bitcoin. Criptomoedas. Portfólio.

\begin{abstract}
With the advance of the cryptocurrencies in particular Bitcoin in relation to population and the valuations of the same mainly in 2017. It becomes common the comparison of Bitcoin with other assets, being used to reduce the risks of an investment portfolio, also being considered a new asset class. In view of this scenario, the following research question is
\end{abstract}


raised: Can Bitcoin benefit from the optimization of an investment portfolio? If yes, what is your participation? The research has a quantitative approach. The research universe comprises 48 monthly observations, in the period between January 2014 and December 2017, for each of the variables used in the study, namely: (a) Ibovespa; (b) IBrX 50; (c) LFT; (d) gold; (e) real-US dollar; and (f) Bitcoin. The calculation procedure for portfolio optimization was done using the Microsoft Excel ${ }^{\circledR}$ Solver tool. A portfolio was set up with and without Bitcoin to answer the research problem. The results showed that portfolio returns are higher in portfolios containing Bitcoin for the years 2015, 2016 and 2017, while in 2014 there were no differences, exactly the year in which Bitcoin's returns were negative. Thus, the addition of Bitcoin in an investment portfolio proved to be advantageous, allowing a better portfolio optimization.

Keywords: Bitcoin. Cryptocurrencies. Portfólio.

\section{Introdução}

A concepção que se tem de dinheiro surgiu com as trocas de mercadorias comumente feitas há muito tempo. A população produzia bens excedentes e precisavam de outros, realizando trocas. Para facilitar esse processo, o dinheiro surgiu como um intermediário, permitindo a venda e aquisição de novos produtos e tornando-se um meio comum de troca (TAN; LOW, 2017). Nesse novo sistema, surgiram, também, os primeiros bancos, que garantiam que o processo funcionasse corretamente. Eles evoluíram e se tornaram os principais influenciadores da economia mundial, controlando a emissão de notas e a concessão de empréstimos e servindo como um intermediário nas transações financeiras. Os governos e os bancos centrais trabalham arduamente para garantir que a moeda fiduciária seja aceita como uma forma de dinheiro e que o fornecimento de dinheiro para a economia não seja inflacionado ou deflacionado (SIMSER, 2015).

Tal poder cedido às instituições financeiras torna-se perigoso, visto que toda a economia fica dependente de suas decisões, o que se torna preocupante, principalmente quando se toma como exemplo a crise de 2008, efetivada por decisões erradas das instituições financeiras (FRANCO; BAZAN, 2018; SIMSER, 2015). Na referida ocasião, a confiança nas instituições despencou, possibilitando o surgimento do Bitcoin (SIMSER, 2015), que busca solucionar as falhas de mercado que apareceram (NAKAMOTO, 2008).

Segundo Nakamoto (2008), é necessário que se tenha um sistema baseado em prova criptográfica e não em confiança, em que seja possível transações envolvendo quaisquer pessoas diretamente, sem a necessidade de um intermediário. Como forma de resolução desse problema, criou-se, o Bitcoin, que é uma moeda digital descentralizada que permite pagamentos globais sem ter uma autoridade central, utilizando o sistema de ponto a ponto, que lhe confere legitimidade (ULRICH, 2014).

A criptografia é a base essencial para que o sistema funcione sem erros. Cada usuário possui duas chaves, uma pública e uma privada. Quando há uma transação, o vendedor acrescenta sua chave privada e insere a chave pública do destinatário. Essa transação é registrada no blockchain, responsável pelo armazenamento de todas as transações e que possibilita a conferência de cada uma dessas movimentações. Existe ainda a figura dos 
mineradores, que nesse sistema são responsáveis pelas validações das transações, evitando fraudes ou gasto duplo de uma única moeda (SIMSER, 2015).

Torna-se comum a comparação do Bitcoin com outros ativos, sendo utilizado para diminuir os riscos. Além disso, como o Bitcoin tem uma negociação diária e constante, consegue uma melhor velocidade nos retornos e garante sua posição no mercado de análise de portfólio e gestão de riscos (DYHRBERG, 2016). É evidente o espaço para as criptomoedas nas carteiras de investimentos, que se apresentam conectados entre si e distantes dos ativos tradicionais. Caracterizam-se como uma nova classe de ativos, possuindo suas características intrínsecas e possibilitando variações aos investidores (CORBET et al., 2018).

O Bitcoin passou a ser encarado não apenas como uma moeda virtual (criptomoeda), mas como uma forma de investimento (BALCILAR et al., 2017; BLAU, 2017; ALVAREZRAMIREZ; RODRIGUEZ; IBARRA-VALDEZ, 2018). Passaram a ser um enfoque de pesquisas possibilidades do uso do Bitcoin como uma forma de busca de melhoria de eficiência de um portfólio de investimento em conjunto com outros ativos considerados tradicionais (WU; PANDEY, 2014; BRIÈRE; OOSTERLINCK; SZAFARZ, 2015; AGGARWAL; SANTOSH; BEDI, 2018; CORBET et al., 2018; KAJTAZI; MORO, 2018; SYMITSI; CHALVATZIS, 2018).

Mesmo que parcela das pesquisas (WU; PANDEY, 2014; BRIÈRE; OOSTERLINCK; SZAFARZ, 2015; AGGARWAL; SANTOSH; BEDI, 2018; CORBET et al., 2018; KAJTAZI; MORO, 2018) sugerem benefícios da inclusão do Bitcoin em uma carteira diversificada, isso não é ponto unanime ainda, conforme apontaram os resultados de Symitsi e Chalvatzis (2018).

Diante dessas lacunas, levanta-se a seguinte questão de pesquisa: $\mathrm{O}$ uso do Bitcoin pode trazer benefícios de otimização de uma carteira de investimentos? Caso sim, qual é a sua participação? Portanto, o objetivo desta pesquisa compreende verificar se o uso do Bitcoin pode trazer benefícios de otimização de uma carteira de investimentos e, caso sim, qual é a sua participação.

Esse levantamento já havia sido desenvolvido em outras localidades, porém havia uma lacuna de interpretação referente a outras moedas que não fossem o dólar. Em um estudo analisando o contexto indiano, Aggarwal, Santosh e Bedi (2018) ressaltam a volatilidade do investimento em Bitcoin e sua rentabilidade, devendo ser incorporado por investidores que não possuem aversão ao risco. Já Kajtazi e Moro (2018) analisaram a construção do portfólio na China, EUA e Europa, concluindo que não há grande divergência no papel do Bitcoin nos contextos estudados. Além disso não existe no contexto brasileiro uma pesquisa que busque a formação de um portfólio considerado os ativos locais com a criptomoedas.

A contribuição da pesquisa avança além do aspecto acadêmico, mas pode permitir que investidores considerem este como uma alternativa de investimentos na formação dos respectivos portfólios, caso seja viável, ou simplesmente descarta-lo.

\section{Fundamentação Teórica}

\subsection{Teoria do portfólio e criptomoedas}

As técnicas de otimização de carteira da média-variância permitem que um investidor encontre a carteira com maior retorno esperado para qualquer nível de variância, dependendo 
do nível de volatilidade que o investidor esteja disposto a aceitar (MARKOWITZ, 1952). A partir desse princípio, o portfólio ideal se caracteriza como uma diversificação de ativos, considerando o retorno esperado e a variação, buscando o menor risco para um nível determinado de retorno desejado (PFIFFELMANN; ROGER; BOURACHNIKOVA, 2016). Trata-se de uma diversificação e maximização dos resultados esperados (MARKOWITZ, 1952).

Mediante a definição do portfólio, torna-se possível a redução parcial do risco, que dependerá da existência ou não de variação conjunta entre os ativos. Para medir a comovimentação dos retornos, são utilizadas duas medidas estatísticas: correlação e covariância (MARKOWITZ, 1952). Se dois grupos de ativos possuem movimentação interligada, seus retornos tenderão a estar ao mesmo tempo abaixo ou acima da média, resultando em uma correlação positiva. Se o contrário ocorrer e a movimentação for em direção oposta, a correlação será negativa, pois um tenderá a estar acima da média enquanto o outro abaixo (BERK; DEMARZO, 2009). É necessário que o investidor evite ativos com alta correlação. Deve-se buscar diferentes ativos de diferentes ramos, com características econômicas diversas, não sofrendo pelos mesmos eventos econômicos e garantindo uma correlação mais baixa (MARKOWITZ, 1952).

Para se definir a força da relação entre os ativos, calcula-se sua correlação entre os retornos, evidenciando riscos comuns e tendências de movimentações juntas. Quanto mais a correlação é próxima de +1 , mais os retornos tendem a se movimentar juntos como consequência de eventos econômicos similares. Quando a correlação é igual a 0, não há tendência para movimentações opostas ou unidas. Finalmente, quando se aproxima de -1 , as movimentações são em direções contrárias (BERK; DEMARZO, 2009).

Formas de avaliar o desempenho de uma carteira de investimento é considerado o retorno, bem como os riscos envolvidos, assim, surgem indicadores que se propõem a fazer essa avaliação, cabendo mencionar o uso do índice de Sharpe, obtido pelo retorno do portfólio já diminuído pela taxa de retorno de ativo livre de risco, dividido pelo desviopadrão da carteira (BERK; DEMARZO, 2009).

Com o advento das criptomoedas, alguns estudos (BRIÈRE; OOSTERLINCK; SZAFARZ, 2015; BOURI et al., 2017b; AGGARWAL; SANTOSH; BEDI, 2018; CORBET et al., 2018; SYMITSI; CHALVATZIS, 2018) começaram a utilizar o Bitcoin como alternativa de investimento, buscando montar um portfólio otimizado com diversos ativos financeiros. Nessas pesquisas, em geral, foram apontadas as baixas correlações do Bitcoin com os demais ativos. Segundo Bouri et al. (2017a), o investimento de curto prazo em Bitcoin pode equilibrar as incertezas do mercado financeiro, especialmente quando se trata de incerteza baixa ou alta, como uma cobertura nos períodos imprecisos da economia global.

Bitcoin é considerado um ativo alternativo, antagônico aos tradicionais ativos financeiros do mercado, tornando-se fonte de refúgio para investidores desacreditados da economia. Com isso, ganha cada vez mais espaço como hedge e diversificador eficaz de carteiras, com constantes presenças de ações, títulos, ouro, commodities e índice de moedas (BOURI et al., 2017b).

De forma similar, Corbet et al. (2018) realizaram um estudo com o uso de ativos, como o índice SP500, e ouro em conjunto com três criptomoedas (Bitcoin, Ripple e Litecoin). Os achados apontaram que as criptomoedas são capazes de proporcionar benefícios de diversificação a um curto prazo de investimentos. 
Segundo estudo realizado por Aggarwal, Santosh e Bedi (2018), o Bitcoin proporciona ganhos de diversificação e melhora significativamente as taxas de risco e retorno. O estudo foi feito em uma carteira de investimentos indianos com seis classes de ativos, e apontou que a volatilidade elevada do Bitcoin o restringe dos investidores que possuem aversão ao risco, mas que é capaz de elevar o retorno de forma consistente e manter sua superioridade em relação a uma carteira sem Bitcoin.

Já Symitsi e Chalvatzis (2018) fizeram uma abordagem com ativos e criptomoedas, explicitando a baixa correlação do Bitcoin com os outros ativos, o que ocasionou uma redução elevada nos riscos do portfólio. E, em oposição com os estudos anteriores, o estudo indicou que o Bitcoin possui pouca contribuição na composição de uma carteira, causando apenas aumento do risco do portfólio como um todo.

Brière, Oosterlinck e Szafarz (2015) utilizaram ações, títulos, moedas, commodities, fundos de hedge e imóveis. A partir desses ativos, fizeram a comparação com o Bitcoin. Dado o período de análise, de 2010 a 2013, a criptomoeda ainda era uma ideia bem recente. Baseados nos fatos históricos, os autores apontaram que ativos ligados a inovações são mais propensos a bolhas. Além disso, o risco de contaminação dos dados coletados do Bitcoin era muito grande, visto que se concentravam em estágio inicial. Outro fato que poderia ser alterado seria a correlação baixa do item com os demais, que costuma aumentar em períodos de crise. Por fim, confirmaram-se os benefícios significativos oferecidos pelo Bitcoin na composição de uma carteira.

De acordo com Kajtazi e Moro (2018), o benefício da inclusão do Bitcoin remete ao ano específico de 2013, visto que foi o maior aumento da volatilidade e valor da criptomoeda. No ano seguinte, essa volatilidade continuou no mesmo padrão, mas sem proporcionar um aumento significativo de valor. Quando adicionado a um portfólio com ativos europeus e outro com ativos chineses, o Bitcoin ocasionou uma melhora, porém estava mais ligado ao aumento de retorno do que à redução da alta volatilidade. Ainda segundo esse pesquisador, não há considerável diferença no papel do Bitcoin nos diferentes contextos estudados, e a criptomoeda pode vir a desempenhar um papel mais destacável na diversificação de carteiras.

\subsection{Tópicos especiais em criptomoedas - blockchain e regulações}

O blockchain consiste em um banco de dados distribuído que garante a segurança de todas as transações envolvendo o Bitcoin. Seu processo se dá por meio de criptografia, e todas as operações são conferidas para que não haja fraude ou gasto duplo da mesma moeda (FANNING; CENTERS, DAVID, 2016). Todos os lançamentos são registrados como numa espécie de livro-razão. Para efetuar as transações, são utilizadas as chaves de segurança, que validarão a movimentação. Para alcançar o anonimato total dos usuários das criptomoedas, essa descentralização é essencial, não permitindo a existência de um controlador central, como é o caso dos bancos (PRYBILA et al., 2017).

Com essa tecnologia, o anonimato do proprietário do Bitcoin é comum, mas com um nível de transparência para cada transação que pode facilmente ser rastreada de volta por meio de todas as transações em que o Bitcoin foi utilizado para o início da sua circulação. Todas as transações de Bitcoin são legíveis por todos nos registros armazenados em uma estrutura de dados amplamente replicada (BÖHME et al., 2015). 
Para garantir o funcionamento e constante verificação do blockchain, o sistema libera enigmas matemáticos que só podem ser solucionados por métodos computacionais intensivos para serem resolvidos pelos chamados mineradores. Ao resolver o enigma apresentado, o usuário publica essa solução no bloco, contendo todas as transações que ocorreram desde a última publicação. Com isso, são verificadas as soluções e garantem total credibilidade às operações. Após a verificação, os mineradores são recompensados em Bitcoins (BÖHME et al., 2015).

A expectativa é conseguir derrubar o uso de intermediários, possibilitando redução do custo das operações. No âmbito mundial, grandes instituições financeiras, como o JP Morgan, já estão tentando substituir o sistema atual pelo blockchain. Porém, essa tecnologia ainda não suporta um grande número simultâneo de operações por vez (LOUREIRO, 2017). Uma das hipóteses pensadas é a criação de um cadastro único entre os bancos, armazenados noblockchain. Com isso, clientes não precisariam fazer o cadastro em cada uma das instituições e ocasionaria uma redução nos processos internos dos bancos (LIMA, 2017).

Em 2014 houve o primeiro debate acerca da regulação das moedas digitais. Guiada pelo superintendente do Departamento de Serviços Financeiros de Nova York, Benjamin Lawsky, foi definido que qualquer negócio que estivesse relacionado a transações de moedas virtuais na cidade deveriam obter a BitLicense, que obrigassem que essas empresas a manter um certo padrão de segurança aos clientes, garantindo a cyber-segurança nacional (DEWAAL; $D E M P S E Y, 2015)$. Segundo seu principal idealizador, a BitLicense fornece proteção aos ativos dos clientes que são mantidos por provedores de serviços, evitando possíveis fraudes. Essa atitude fez com que empreendedores deixassem de lado seus negócios, visto que o custo para se operar em Nova York e ainda conseguir lucrar se apresentava cada vez mais elevado (CANNUCCIARI, 2017). Por outro lado, evidencia-se o reconhecimento do valor a longo prazo das moedas virtuais por parte das entidades governamentais dos Estados Unidos da América (EUA), desenvolvendo um regulamento que possibilita o desenvolvimento e aumento de segurança para seus investidores (GREEBEL et al., 2015). Entidades que precisam relatar em suas demonstrações financeiras esse tipo de transação são descritas a seguir: (a) atividade de compra e venda de moedas virtuais; (b) atividade de prestação de trocas de moedas; e (c) atividade de mineração (RADU; ISAI; MIHAI, 2017).

O blockchain se apresenta ainda como uma inovação muito nova, que necessita de obstáculos que possibilitam o desenvolvimento de seu potencial. Regulações e imposições governamentais afetam diretamente a velocidade desse crescimento, cedendo ao governo a análise de contribuição dessa nova tecnologia para o atual sistema financeiro (YEOH, 2017).

Cabe ressaltar que no Brasil não existe, até o momento, regras e regulações claras a respeito do uso do Bitcoin e/ou demais criptomoedas em geral, seja como moeda virtual e/ ou investimento. A exceção a essa situação é mediante posicionamentos do Banco Central do Brasil (Bacen) e da Comissão de Valores Mobiliários (CVM). O Bacen (2017, p. 1), por meio do Comunicado n⿳ 31.379/2017, afirma que as moedas virtuais "não são emitidas nem garantidas por qualquer autoridade monetária". Já a CVM emitiu um comunicado vedando aos fundos de investimento alocarem recursos em criptomoedas, inclusive o Bitcoin, $\mathrm{o}$ posicionamento é de caráter temporário e menciona o Projeto de Lei no 2.303/2015, que busca restringir as aplicações nas criptomoedas (ALVARENGA, 2018). 


\subsection{Criptomoedas e investimentos}

A ideia de criptomoeda surgiu com os cypherpunks e seus estudos sobre criptografia, buscando inicialmente garantir sua privacidade financeira e liberdade. Antes do Bitcoin, muitas moedas foram idealizadas, mas eram silenciadas por organizações financeiras ou deixavam lacunas em sua utilização, como o problema do gasto duplo (CANNUCCIARI, 2017; FRANCO; BAZAN, 2018). Atualmente, o Bitcoin se classifica como um ativo da economia alternativa, opondo-se às moedas tradicionais. Ele pode atuar como um hedge e seguro contra movimentos altamente voláteis, como os presentes em investimentos em ações, além de garantir retornos mais elevados em curto prazo (BOURI et al., 2017b). Por outro lado, sua superioridade tecnológica e sua redução de custos transacionais se tornam altamente chamativas. Trata-se de um bem escasso, com quantidade já delimitada de moedas, que vem conquistando cada vez mais adeptos (ULRICH, 2016). Bitcoin é de interesse para os economistas como uma moeda virtual com potencial para perturbar os sistemas de pagamento existentes e talvez até mesmo os sistemas monetários completos, visto que traz soluções para falhas comprovadas do atual sistema financeiro. Mesmo em sua fase inicial atual, tais moedas virtuais já forneciam uma variedade de ideias sobre o design de mercado e do comportamento de compradores e vendedores (BÖHME et al., 2015).

$\mathrm{O}$ uso das criptomoedas proporciona às empresas o acesso potencial a novas tecnologias, mas também expõe a empresa a riscos adicionais, dentre eles, estão: volatilidade do preço, futura legislação, roubo ou perda e confiabilidade de terceiros (GRANT; HOGAN, 2015). Quanto a estudos envolvendo a volatilidade, percebe-se que os investidores são atraídos após grande taxa de volatilidade e volumes de negociação (URQUHART, 2018), bem como utilizam a memória estimada para capturar lucros especulativos (CHEAH et al., 2018). Já para as situações de roubo e/ou perda da criptomoeda, em que o investidor não consegue rastreá-la nem possui à sua disposição qualquer mecanismo para recuperá-la, percebe-se que, embora o anonimato seja defendido como uma forma de privacidade para os clientes, ocasiona riscos maiores (DOSTOV; SHUST, 2014).

Mesmo sendo difícil encontrar provas concretas de uso em grande escala de Bitcoins e/ou outras criptomoedas por grupos terroristas e seus partidários, há fortes evidências para sugerir que ele foi utilizado como forma de transferência de recursos para financiar uma série de ataques terroristas na Europa e na Indonésia. As principais organizações terroristas veem no Bitcoin uma forma para diminuir os riscos que envolvem transferências tradicionais de dinheiro. Inúmeras doações são recebidas por sítios associados para financiar esses ataques, exigindo uma investigação que aponte formas viáveis de diferenciar essas transações (IRWIN; MILAD, 2016).

As empresas que optam pelo uso do Bitcoin devem tomar precauções e agir com cautela. Algumas das características vantajosas da criptomoeda que contribuem para seu sucesso são, ao mesmo tempo, lacunas que dificultam os processos de monitoramento. No entanto, com a facilidade que proporciona aos usuários, muitas empresas estão adotando o Bitcoin como forma alternativa de pagamento (GRANT; HOGAN, 2015).

Segundo Symitsi e Chalvatzis (2018), ao longo do tempo, as contribuições destacáveis alcançadas pela criptomoeda são reduzidas pelo aumento dos custos nas transações. A alta volatilidade desse ativo em específico faz com que os investidores se sintam mais atentos diante do risco de ataques cibernéticos, possíveis regulamentos ou restrições. 
No Brasil, uma prova da rápida popularização do Bitcoin é que, em pouco tempo de existência, as exchanges (corretoras para compra e venda de criptomoedas) já possuem o dobro de investidores cadastrados em relação à bolsa de valores brasileira. A previsão é que esse número logo ultrapasse o Tesouro Direto, que já conta hoje com cerca de 1,8 milhões de usuários. O Bitcoin virou febre após sua cotação chegar a US\$ 20 mil em 2017, com uma valorização de 1.400\% só em 2017 (ESTADÃO, 2018).

Em relação aos investimentos tradicionais, se comparados com o Bitcoin, cabe destacar algumas formas de investimento que, segundo a teoria do portfólio, podem ser consideradas na busca de uma carteira com um desempenho otimizado e que são utilizadas na presente pesquisa: (a) Ibovespa; (b) IBrX 50; (c) LFT; (d) ouro; e (e) real-dólar.

O Índice Bovespa (Ibovespa) se caracteriza como o principal indicador médio das ações listadas na Bolsa de Valores, Mercadorias e Futuros de São Paulo (BM\&FBOVESPA, 2018a), como resultado de uma carteira teórica de ativos. Já o IBrX 50 é composto pelos 50 ativos de maior representatividade e negociabilidade nacional, representando um indicador de desempenho médio das principais ações (BM\&FBOVESPA, 2018b).

O Tesouro Direto é um programa desenvolvido pelo Tesouro Nacional, em conjunto com a Companhia Brasileira de Liquidação e Custódia (CBLC), visando possibilitar a venda de títulos públicos a pessoas físicas por meio da Internet (TESOURO NACIONAL, 2018a). Um dos títulos públicos ofertados é a Letra Financeira do Tesouro (LFT), que possui rentabilidade pós-fixada, seguindo a variação da taxa referencial do Sistema Especial de Liquidação e de Custódia (Selic), considerada a taxa básica de juros da economia nacional (TESOURO NACIONAL, 2018b).

Quanto ao ouro, historicamente, oferece segurança, proteção e rentabilidade e atrai diversos investidores, em todo o mundo (BM\&FBOVESPA, 2018c). Quando se opta pela compra do ouro, o investidor precisa escolher uma corretora que vai indicá-lo um fundidor, que deve ser credenciado e autorizado pela bolsa de valores, fornecendo-lhe as barras de ouro. Nessa operação, são envolvidas diversas taxas, como: custódia, corretagem, seguro transporte e Imposto de Renda (IR) em aplicações mensais superiores a $\mathrm{R}$ \$ 20 mil. É vinculada às cotações da London Stock Exchange e da New York Stock Exchange, além de sofrer influências diretas do dólar e das leis de oferta e procura (BM\&FBOVESPA, 2018c).

Por último, o uso da cotação da moeda do dólar dos EUA frente ao real se apresenta como uma alternativa de proteção contra as desvalorizações da moeda brasileira e de ganhos financeiros adicionais.

\section{Metodologia}

\subsection{Seleção da amostra e coleta de dados}

O universo da pesquisa compreende 48 observações mensais, no período entre janeiro de 2014 a dezembro de 2017, para cada uma das variáveis utilizadas no estudo, a saber: (a) Ibovespa; (b) IBrX 50; (c) LFT; (d) ouro; (e) real-dólar; e (f) Bitcoin. Os ativos escolhidos coincidem com os utilizados nos trabalhos desenvolvidos por Wu e Pandey (2014), Brière, Oosterlinck e Szafarz (2015) e Symitsi e Chalvatzis (2018).

A escolha do período para investigação se deu em virtude da limitação de acesso

aos dados do Bitcoin, visto que os preços de fechamento compreendendo um ano de forma 
integral estão disponíveis a partir de 2014 na base de dados Economatica ${ }^{\circledR}$, as cotações extraídas se referem à exchange brasileira Mercado Bitcoin. Os dados das demais variáveis também foram coletados da base de dados Economatica ${ }^{\circledR}$.

\subsection{Tratamento dos dados e procedimentos}

Com base nos dados coletados, foram feitos os cálculos dos retornos de cada um dos ativos considerados, bem como os desvios-padrão, cálculo das correlações e covariâncias que serviram de insumos para a formulação da carteira.

Para que fosse possível uma análise mais detalhada, fez-se uma análise do período global de janeiro de 2014 a dezembro de 2017, correspondendo a quatro anos, bem como a composição da carteira para cada ano de forma isolada, para que fosse possível identificar possíveis tendências e a vantagem ou não da adoção do Bitcoin em uma carteira de investimentos de forma mais consistente.

Para a composição da carteira ótima para cada período de análise, foram inicialmente considerados pesos idênticos de participação para cada um dos ativos considerados na pesquisa. Dessa forma, chegou-se aos valores de retornos e desvios-padrão das carteiras. Em seguida, o procedimento de cálculo para otimização da carteira se deu mediante o uso da ferramenta Solver do Microsoft Excel ${ }^{\circledR}$, em que se estabeleceu como condição a situação em que o desvio-padrão da carteira ótima não pode ultrapassar o desvio-padrão da carteira que continha pesos igualmente distribuídos.

Após essa condição, estabeleceu-se que a carteira pode assumir qualquer composição que maximize o seu retorno.

Com os critérios atendidos, chegou-se a resultados de composição para cada ano, em que se tem carteiras com riscos (desvios-padrão) controlados, evitando desvios de cálculos que buscassem a maximização do retorno sem a devida preocupação com o controle dos riscos.

Em seguida, para cada carteira, foi calculado o índice de Sharpe, para que existisse uma forma crível de comparação entre as carteiras ao longo dos anos, bem como comparar com o teste adicional feito ao se montar carteiras sem a possibilidade do uso do Bitcoin e outras com a possibilidade do uso deste, permitindo, assim, responder ao problema de pesquisa proposto. Para o índice de Sharpe, necessita-se do uso de uma taxa de retorno de um ativo livre de risco para diminuir dos retornos considerados nos demais ativos, para isso, optou-se pela taxa de retorno da LFT.

\section{Resultados}

Para analisar o comportamento do Bitcoin, foi feito um comparativo com outros ativos financeiros e suas propriedades evidenciando sua capacidade de retorno. Na Tabela 1, são demonstrados os resultados médios mensais de 2014 a 2017. 
Tabela 1 - Estatística descritiva dos retornos médios mensais de 2014 a 2017

\begin{tabular}{lcccc}
\hline & Mínimo & Máximo & Média & Desvio-padrão \\
\hline Bitcoin & $-24,33 \%$ & $115,37 \%$ & $10,24 \%$ & $29,62 \%$ \\
Ibovespa & $-11,70 \%$ & $16,97 \%$ & $1,02 \%$ & $6,36 \%$ \\
IBrX 50 & $-11,54 \%$ & $16,41 \%$ & $0,99 \%$ & $6,25 \%$ \\
LFT & $0,51 \%$ & $1,18 \%$ & $0,95 \%$ & $0,17 \%$ \\
Ouro & $-9,35 \%$ & $11,48 \%$ & $0,94 \%$ & $4,56 \%$ \\
Real-dólar & $-11,05 \%$ & $11,74 \%$ & $0,82 \%$ & $4,63 \%$ \\
\hline
\end{tabular}

Fonte: Dados da pesquisa.

Na Tabela 1 percebe-se a grande disparidade do Bitcoin com os outros ativos, ao se analisar a média do retorno mensal e o desvio-padrão ao longo dos quatro anos, sendo resultado da alta volatilidade que é inerente à criptomoeda. Quanto ao alto desvio-padrão, evidencia o risco e volatilidade que envolvem o investimento.

Logo após, aparece o Ibovespa e o IBrX 50 como representantes do mercado de ações, que comumente apresentam variação constante e um desvio mais elevado que os demais. A LFT se comporta de forma peculiar, sendo a única a não possuir variações positivas e negativas, estabilidade típica de títulos públicos que buscam manter o índice de rendimento ao longo dos anos.

Na Tabela 2, são demonstrados os resultados médios mensais segregados por ano e ativo correspondente, bem como o período de 2014 a 2017 de forma consolidada.

Tabela 2 - Retorno médio mensal por ano

\begin{tabular}{lccccc}
\hline & 2014 & 2015 & 2016 & 2017 & $2014-2017$ \\
\hline Bitcoin & $-4,97 \%$ & $7,42 \%$ & $6,53 \%$ & $31,97 \%$ & $10,24 \%$ \\
Ibovespa & $-0,05 \%$ & $-1,03 \%$ & $3,08 \%$ & $2,08 \%$ & $1,02 \%$ \\
IBrX 50 & $-0,02 \%$ & $-1,02 \%$ & $2,94 \%$ & $2,07 \%$ & $0,99 \%$ \\
LFT & $0,87 \%$ & $1,04 \%$ & $1,10 \%$ & $0,79 \%$ & $0,95 \%$ \\
Ouro & $1,00 \%$ & $2,59 \%$ & $-0,94 \%$ & $1,11 \%$ & $0,94 \%$ \\
Real-dólar & $1,06 \%$ & $3,49 \%$ & $-1,48 \%$ & $0,19 \%$ & $0,82 \%$ \\
\hline
\end{tabular}

Fonte: Dados da pesquisa.

No ano de 2014, o Bitcoin apresentou uma queda significativa em seu retorno, após o escândalo de hackers na Mt Gox, grandes especialistas no câmbio da moeda, alarmavam do risco aos seus usuários, que já estavam abalados pela propagação do uso do Bitcoin para fins ilícitos na Deep Web no ano anterior (CANNUCCIARI, 2017).

Após isso, percebeu-se uma expressiva ascensão logo no ano seguinte. No ano de 2017, apresentou sua maior taxa de retorno (CHAVEZ-DREYFUSS, 2017) e garantiu um retorno superior a qualquer outro ativo, confirmando os achados de Aggarwal, Santosh e Bedi (2018).

O Ibovespa e o IBrX 50 apresentam quedas consecutivas em 2014 e 2015, que podem ser justificadas com a conjuntura econômica nacional, que passava por instabilidades, fazendo com que investidores procurassem outros investimentos que lhe proporcionassem menor risco.

Em 2016, o retorno voltou a ficar positivo, com a expectativa de reaquecimento da economia e prospecção das ações brasileiras (MORAES, 2016).

Na Tabela 3, são demonstrados os desvios-padrão médios mensais por ano. 
Tabela 3 - Desvio-padrão médio mensal por ano

\begin{tabular}{|c|c|c|c|c|c|}
\hline & 2014 & 2015 & 2016 & 2017 & 2014-2017 \\
\hline Bitcoin & $15,18 \%$ & $15,99 \%$ & $13,88 \%$ & $47,73 \%$ & $29,62 \%$ \\
\hline Ibovespa & $6,47 \%$ & $5,90 \%$ & $8,21 \%$ & $4,11 \%$ & $6,36 \%$ \\
\hline IBrX 50 & $6,55 \%$ & $5,68 \%$ & $8,05 \%$ & $4,02 \%$ & $6,25 \%$ \\
\hline LFT & $0,06 \%$ & $0,10 \%$ & $0,06 \%$ & $0,19 \%$ & $0,17 \%$ \\
\hline Ouro & $3,35 \%$ & $5,69 \%$ & $5,84 \%$ & $2,08 \%$ & $4,56 \%$ \\
\hline Real-dólar & $3,56 \%$ & $5,54 \%$ & $5,28 \%$ & $2,54 \%$ & $4,63 \%$ \\
\hline
\end{tabular}

Fonte: Dados da pesquisa.

Em todo o período analisado, o Bitcoin apresentou os maiores índices de risco em consequência da sua alta volatilidade, principalmente no ano de 2017, quando apresentou seu maior crescimento em termos de valor do ativo, alcançando números exorbitantes e inéditos para as criptomoedas (CHAVEZ-DREYFUSS, 2017). Em contrapartida, as LTFs se comportam como um hedge para os investidores, apresentando baixas taxas de desviopadrão, característica comum aos títulos públicos.

Já a Tabela 4 evidencia a correlação existente entre os ativos financeiros baseados nos dados mensais de 2014 a 2017.

Tabela 4 - Matriz de correlação com base nos dados mensais de 2014 a 2017

\begin{tabular}{lcccccc}
\hline & Bitcoin & Ibovespa & IBrX 50 & LFT & Ouro & Real-dólar \\
\hline Bitcoin & 1 & 1 & & & \\
Ibovespa & $-0,043$ & $0,999^{*}$ & 1 & & & \\
IBrX 50 & $-0,056$ & $-0,092$ & $-0,098$ & 1 & & \\
LFT & $-0,220$ & $-0,396^{*}$ & $-0,390^{*}$ & $-0,042$ & 1 & 1 \\
Ouro & $-0,025$ & $-0,594^{*}$ & $-0,591^{*}$ & $-0,016$ & $0,574^{*}$ & \\
Real-dólar & 0,076 & * Correlação significativa no nível de 1\%. &
\end{tabular}

Fonte: Dados da pesquisa.

Corroborando os estudos de Aggarwa, Santosh e Bedi (2018), o Bitcoin apresenta correlação negativa com grande parte dos ativos analisados, confirmando sua característica de diversificador de portfólios. As pesquisas de Wu e Pandey (2014), Brière, Oosterlinck e Szafarz (2015) e Symitsi e Chalvatzis (2018) apontam uma baixa ou insignificante correlação da criptomoeda com os demais itens.

Nota-se como maior correlação Ibovespa e IBrX 50, o que já era esperado, pelo fato de que o IBrX 50 é uma fragmentação das empresas contidas no Ibovespa, sendo diretamente correlacionadas. O ouro apresenta correlação oposta com o Ibovespa e IBrX 50, por se tratarem de formas alternativas de investimento em períodos diversos da economia. Quando ela passa por instabilidade, eleva-se a migração de recursos para investimentos como o ouro e, em momentos de aquecimento econômico, buscam-se ações com índices de rentabilidade maiores.

Percebe-se que, com exceção do real-dólar e Ibovespa/IBrX 50, as demais relações se comportam de formas opostas, com correlações baixas e sem possíveis alterações pelos mesmos eventos econômicos.

Na Tabela 5, buscou-se montar uma carteira ideal e demonstrar a porcentagem presente de cada item, sem considerar o Bitcoin. 
Tabela 5 - Formação do portfólio ótimo sem o Bitcoin

\begin{tabular}{|c|c|c|c|c|c|}
\hline \multirow{2}{*}{ Ativos } & \multicolumn{5}{|c|}{ Portfólio } \\
\hline & 2014 & 2015 & 2016 & 2017 & 2014-2017 \\
\hline Ibovespa & $0,00 \%$ & $0,00 \%$ & $34,01 \%$ & $0,00 \%$ & $35,59 \%$ \\
\hline IBrX 50 & $0,00 \%$ & $0,00 \%$ & $0,00 \%$ & $39,56 \%$ & $0,00 \%$ \\
\hline LFT & $42,21 \%$ & $61,19 \%$ & $65,99 \%$ & $44,41 \%$ & $52,83 \%$ \\
\hline Ouro & $17,95 \%$ & $0,00 \%$ & $0,00 \%$ & $16,02 \%$ & $11,58 \%$ \\
\hline Real-dólar & $39,84 \%$ & $38,81 \%$ & $0,00 \%$ & $0,00 \%$ & $0,00 \%$ \\
\hline Retorno da carteira & $0,10 \%$ & $0,95 \%$ & $0,67 \%$ & $0,56 \%$ & $0,02 \%$ \\
\hline Desvio-padrão da carteira & $1,71 \%$ & $2,07 \%$ & $2,68 \%$ & $1,64 \%$ & $2,08 \%$ \\
\hline Índice de Sharpe & $6,07 \%$ & $45,91 \%$ & $25,15 \%$ & $33,79 \%$ & $1,14 \%$ \\
\hline
\end{tabular}

Fonte: Dados da pesquisa.

Em 2014, o principal componente se tornou a LFT, título atrativo e incentivado no período deficitário para arrecadação de recursos financeiros. Quanto às ações, são excluídas por apresentarem desvios mais elevados, representando maior risco no período.

Já em 2015 o ouro perdeu posição, podendo ser justificado pelo aumento na taxa de adesão à LFT, havendo uma possível migração nos recursos. A LFT continua a apresentar alta taxa de retorno, comparada ao baixo risco que oferece, e o real perde mercado, devido à crescente valorização do dólar, que também afetou a carteira de 2017.

A partir de 2017, ativos como IBrX 50 ganharam destaque. A LFT apresentou queda significativa por estar ligada à Selic, que sofreu declínio também em 2017, fazendo com que o investimento se tornasse menos rentável, porém não deixando de ser utilizada pelos investidores mais conservadores, que preferem não se arriscar na bolsa de valores. $\mathrm{O}$ ouro voltou a se destacar, por ser considerado um ativo seguro de investimento e não sofrer oscilações da inflação e da própria economia nacional.

A Tabela 6 demonstra a composição de um portfólio eficiente com a presença do Bitcoin e como os itens seriam alocados para garantir ao investidor o maior retorno e menor risco possível.

Tabela 6 - Formação do portfólio ótimo com o Bitcoin

\begin{tabular}{|c|c|c|c|c|c|}
\hline \multirow{2}{*}{ Ativos } & \multicolumn{5}{|c|}{ Portfólio } \\
\hline & 2014 & 2015 & 2016 & 2017 & $2014-2017$ \\
\hline Bitcoin & $0,00 \%$ & $15,15 \%$ & $16,26 \%$ & $17,47 \%$ & $18,27 \%$ \\
\hline Ibovespa & $0,00 \%$ & $0,00 \%$ & $21,07 \%$ & $0,00 \%$ & $2,08 \%$ \\
\hline IBrX 50 & $0,00 \%$ & $0,00 \%$ & $0,00 \%$ & $82,53 \%$ & $0,00 \%$ \\
\hline LFT & $0,09 \%$ & $39,57 \%$ & $62,67 \%$ & $0,00 \%$ & $79,65 \%$ \\
\hline Ouro & $30,85 \%$ & $24,13 \%$ & $0,00 \%$ & $0,00 \%$ & $0,00 \%$ \\
\hline Real-dólar & $69,05 \%$ & $21,15 \%$ & $0,00 \%$ & $0,00 \%$ & $0,00 \%$ \\
\hline Retorno da carteira & $0,18 \%$ & $1,86 \%$ & $1,30 \%$ & $6,50 \%$ & $1,70 \%$ \\
\hline Desvio-padrão da carteira & $2,94 \%$ & $3,18 \%$ & $2,29 \%$ & $7,55 \%$ & $5,07 \%$ \\
\hline Índice de Sharpe & $6,12 \%$ & $58,35 \%$ & $56,85 \%$ & $86,04 \%$ & $33,50 \%$ \\
\hline
\end{tabular}

Fonte: Dados da pesquisa.

A Tabela 6 apresenta a formação do portfólio ideal com o Bitcoin. No primeiro ano simulado, chegou-se no índice de $0 \%$ para investimentos em Bitcoin após analisar a taxa de retorno, que ficou negativa nesse período. Nos anos seguintes, passou a haver a presença constante do ativo, porém em um percentual controlado, representando entre 15,15\% e $17,47 \%$ dos investimentos totais, dada a sua grande volatilidade e risco. Em contrapartida, os investimentos em ouro e moeda nacional despencaram ao longo dos anos. Os demais ativos comportaram-se de forma similar à exposta na Tabela 5 .

Por fim, a Tabela 7 traz um comparativo dos índices resultantes das carteiras com e sem o Bitcoin, abordando seu retorno, o desvio-padrão e o índice Sharpe. 
Tabela 7 - Comparativo das métricas dos portfólios com e sem o Bitcoin

\begin{tabular}{|c|c|c|c|c|c|c|}
\hline \multirow{2}{*}{ Período } & \multicolumn{2}{|c|}{ Retorno da carteira } & \multicolumn{2}{|c|}{ Desvio-padrão da carteira } & \multicolumn{2}{|c|}{ Índice de Sharpe } \\
\hline & Com Bitcoin & Sem Bitcoin & Com Bitcoin & Sem Bitcoin & Com Bitcoin & Sem Bitcoin \\
\hline 2014 & $0,18 \%$ & $0,10 \%$ & $2,94 \%$ & $1,71 \%$ & $6,12 \%$ & $6,07 \%$ \\
\hline 2015 & $1,86 \%$ & $0,95 \%$ & $3,18 \%$ & $2,07 \%$ & $58,35 \%$ & $45,91 \%$ \\
\hline 2016 & $1,30 \%$ & $0,67 \%$ & $2,29 \%$ & $2,68 \%$ & $56,85 \%$ & $25,15 \%$ \\
\hline 2017 & $6,50 \%$ & $0,56 \%$ & $7,55 \%$ & $1,64 \%$ & $86,04 \%$ & $33,79 \%$ \\
\hline $2014-2017$ & $1,70 \%$ & $0,02 \%$ & $5,07 \%$ & $2,08 \%$ & $33,50 \%$ & $1,14 \%$ \\
\hline
\end{tabular}

Fonte: Dados da pesquisa.

Mediante esses dados, evidencia-se que os retornos dos portfólios são maiores em carteiras contendo o Bitcoin, chegando a oferecer uma diferença de quase 6\% em 2017 e tendo como menor variação o ano de 2014, uma vez que o Bitcoin apresentou retorno negativo na época e sequer foi incluído na carteira.

Quanto ao desvio-padrão da carteira, com exceção de 2016, todos os demais oferecem um desvio consideravelmente maior com a presença do Bitcoin, com risco mais elevado, como já analisado com o próprio ativo separadamente.

Para que fosse possível permitir uma melhor comparação entre os resultados dos retornos e desvios-padrão das carteiras com e sem o Bitcoin, o índice Sharpe, contorna esta situação, visto que pondera os retornos pelo risco. E assim as carteiras que possuem maior índice tendem a ser as melhores para implementação. Dessa forma, os resultados do índice de Sharpe consolidam a eficiência da presença do Bitcoin em carteiras ideais, aprimorando o desempenho dos portfólios e garantindo ao investidor maior retorno em um mesmo período, seja ele nos portfólios anuais ou o período de quatro anos.

Os anos de 2015, 2016 e 2017 apresentaram resultados discrepantes no índice Sharpe, a favor da carteira com o Bitcoin, curiosamente a carteira de 2014 que teve diferença praticamente inexistente foi o ano em que o Bitcoin não se mostrou interessante para composição da carteira, obviamente o ano de retorno negativo do Bitcoin.

\section{Considerações Finais}

Nos últimos quatro anos, o Bitcoin vem se destacando como forma de investimento e atraindo pessoas que buscam maior rentabilidade e não possuem aversão ao risco (FRANCO; BAZAN, 2018). Essa realidade faz surgir a necessidade de análises quanto ao comportamento do criptoativo e suas possíveis relações com os demais itens financeiros e que permitam uma formação de um portfólio de investimento. Trabalhos desenvolvidos por Wu e Pandey (2014), Brière, Oosterlinck e Szafarz (2015) e Symitsi e Chalvatzis (2018), testaram o uso do Bitcoin em portfólios de investimento com outros ativos. Diante desse contexto o trabalho teve como objetivo verificar se o uso do Bitcoin pode trazer benefícios de otimização de uma carteira de investimentos e, caso sim, qual é a sua participação.

Mediante aplicação do uso da ferramenta Solver do Microsoft Excel ${ }^{\circledR}$, e com dados compreendidos de 2014 a 2017, os resultados em geral apontaram que o acréscimo do Bitcoin em um portfólio de investimento se mostrou vantajosa, assim apontando que este permite trazer uma melhor otimização da carteira, quanto ao peso de participação, excluindo o ano de 2014, foram: de 15,15\%, em 2015; 16,26\% em 2016; 17,47\% em 2017; por ultimo ao considerar o período global de 2014 a 2017 a carteira deveria possuir 18,27\%. A percepção 
de que as carteias com o Bitcoin foi melhor é comprovada pelo índice Sharpe, que em todos os anos analisados foi superior nos portfólios com a criptomoeda.

Assim estes resultados corroboram os achados de pesquisas anteriores (WU; PANDEY, 2014; BRIÈRE; OOSTERLINCK; SZAFARZ, 2015; AGGARWAL; SANTOSH; BEDI, 2018; CORBET et al., 2018; KAJTAZI; MORO, 2018) que indicaram benefícios da inclusão do Bitcoin em um portfólio diversificado, e indo de encontro aos resultados de Symitsi e Chalvatzis (2018).

Por meio da análise empírica dos dados, percebe-se que a presença do Bitcoin nos portfólios afeta diretamente o retorno e o risco que o investidor terá, porém é essencial para garantir a eficiência da carteira. Cabe ao investidor ponderar sobre o mercado e a economia em que está inserido, podendo optar por maior segurança ou maiores retornos, assumindo o risco necessário.

Como limitações da pesquisa, deve se considerar que não foram testados alguns ativos apenas, além deles estarem ligados a economia brasileira, o uso dos dados dos retornos do Bitcoin, foi de uma Exchange que opera no Brasil, e que aspectos distintos do que ocorre em mercados desenvolvidos podem não se repetir. Além de aspectos de tempo de análise que apesar de ser abrangente o Bticoin é algo muito novo e que carece de mais estudos. Para sugestões futuras, podem ser feitos estudos que considerem o Bitcoin, como uma alternativa de hedge seguro, dentro do mercado brasileiro.

\section{Referências}

AGGARWAL, S.; SANTOSH, M.; BEDI, P. Bitcoin and Portfolio Diversification: Evidence from India. Cham: Springer, 2018.

ALVARENGA, D. CVM proíbe fundos de investir em Bitcoin e outras criptomoedas.

Disponível em: <https://g1.globo.com/economia/noticia/cvm-proibe-fundos-de-investirem-criptomoedas.ghtml>. Acesso em: 15 jan. 2018.

ALVAREZ-RAMIREZ, J.; RODRIGUEZ, E.; IBARRA-VALDEZ, C. Long-range correlations and asymmetry in the Bitcoin market. Physica A: Statistical Mechanics and its Applications, v. 492, p. 949-955, 2018.

BACEN. Comunicado 31.379, de 16 de novembro de 2017Brasil, 2017. . Disponível em: <http://www.bcb.gov.br/pre/normativos/busca/normativo. asp?numero=31379\&tipo=Comunicado\&data $=16 / 11 / 2017>$.

BALCILAR, M.; BOURI, E.; GUPTA, R.; ROUBAUD, D. Can volume predict Bitcoin returns and volatility? A quantiles-based approach. Economic Modelling, v. 64, p. 74-81, 2017.

BERK, J.; DEMARZO, P. Finanças Empresariais. Porto Alegre: Bookman, 2009.

BLAU, B. M. Price dynamics and speculative trading in Bitcoin. Research in International Business and Finance, v. 41, p. 493-499, 2017. 
BM\&FBOVESPA. Índice Bovespa. Disponível em: <http://www.bmfbovespa.com.br/pt_br/ produtos/indices/indices-amplos/indice-bovespa-ibovespa.htm>. Acesso em: 23 jun. 2018a.

BM\&FBOVESPA. Índice Brasil 50 - IBrX-50. Disponível em: <http://www.bmfbovespa.com. br/pt_br/produtos/indices/indices-amplos/indice-brasil-50-ibrx-50.htm>. Acesso em: 23 jun. 2018b.

BM\&FBOVESPA. Futuro de Ouro. Disponível em: <http://www.bmfbovespa.com.br/pt_br/ produtos/listados-a-vista-e-derivativos/commodities/futuro-de-ouro.htm>. Acesso em: 23 jun. 2018c.

BÖHME, R.; CHRISTIN, N.; EDELMAN, B.; MOORE, T. Bitcoin: Economics, Technology, and Governance. Journal of Economic Perspectives, v. 29, n. 2, p. 213-238, 2015.

BOURI, E.; GUPTA, R.; TIWARI, A. K.; ROUBAUD, D. Does Bitcoin hedge global uncertainty? Evidence from wavelet-based quantile-in-quantile regressions. Finance Research Letters, v. 23, p. 87-95, 2017a.

BOURI, E.; MOLNÁR, P.; AZZI, G.; ROUBAUD, D.; HAGFORS, L. I. On the hedge and safe haven properties of Bitcoin: Is it really more than a diversifier? Finance Research Letters, $v$. 20, p. 192-198, $2017 b$.

BRIÈRE, M.; OOSTERLINCK, K.; SZAFARZ, A. Virtual currency, tangible return: Portfolio diversification with bitcoin. Journal of Asset Management, v. 16, n. 6, p. 365-373, 2015.

CANNUCCIARI, C. Banking on Bitcoin. Estados Unidos da América. Gravitas Ventures, 2017. Disponível em: <https://www.netflix.com/br/title/80154500>.

CHAVEZ-DREYFUSS, G. Valor da bitcoin sobe 10 vezes em 2017 e ultrapassa US\$ 10 mil. Disponível em: <https:/economia.uol.com.br/noticias/reuters/2017/11/29/valor-da-bitcoinsobe-10-vezes-em-2017-e-ultrapassa-us-10-mil.htm>. Acesso em: 7 jul. 2018.

CHEAH, E.-T.; MISHRA, T.; PARHI, M.; ZHANG, Z. Long memory interdependency and inefficiency in Bitcoin markets. Economics Letters, 2018.

CORBET, S.; MEEGAN, A.; LARKIN, C.; LUCEY, B.; YAROVAYA, L. Exploring the Dynamic Relationships between Cryptocurrencies and Other Financial Assets. Economics Letters, v. 165, p. 28-34, 2018.

DEWAAL, G.; DEMPSEY, G. New York BitLicense regulations virtually certain to significantly impact transactions in virtual currencies. Journal of Investment Compliance, v. 16, n. 4, p. 59-65, 2015.

DOSTOV, V.; SHUST, P. Cryptocurrencies: an unconventional challenge to the AML/CFT regulators? Journal of Financial Crime, v. 21, n. 3, p. 249-263, 2014.

DYHRBERG, A. H. Hedging capabilities of bitcoin. Is it the virtual gold? Finance Research Letters, v. 16, p. 139-144, 2016. 
ESTADÃO. Número de Investidores de Bitcoins “Encosta” no Total do Tesouro Direto. Disponível em: <https://revistapegn.globo.com/Tecnologia/noticia/2018/02/numero-deinvestidores-de-bitcoins-encosta-no-total-do-tesouro-direto.html\#>. Acesso em: 16 fev. 2018.

FANNING, K.; CENTERS, DAVID, P. Blockchain and Its Coming Impact on Financial Services. The Journal of Corporate Accounting \& Finance, v. 27, n. 5, p. 53-57, 2016.

FRANCO, A.; BAZAN, V. Criptomoedas: Melhor que Dinheiro. São Paulo: Empiricus, 2018.

GRANT, G.; HOGAN, R. Bitcoin: Risks and Controls. The Journal of Corporate Accounting \& Finance, v. 26, n. 5, p. 29-35, 2015.

GREEBEL, E. L.; MORIARTY, K.; CALLAWAY, C.; XETHALIS, G. Recent key Bitcoin and virtual currency regulatory and law enforcement developments. Journal of Investment Compliance, v. 16, n. 1, p. 13-18, 2015.

IRWIN, A. S. M.; MILAD, G. The use of crypto-currencies in funding violent jihad. Journal of Money Laundering Control, v. 19, n. 4, p. 407-425, 2016.

KAJTAZI, A.; MORO, A. Bitcoin and Portfolio Diversification: Evidence from Portfolios of U.S., European and Chinese Assets. SSRN Electronic Journal, p. 1-44, 2018. Disponível em: <http://dx.doi.org/10.2139/ssrn.3095172>. Acesso em: 9 jul. 2018.

LIMA, M. Bancos brasileiros usarão dados reais em testes de blockchain em 2018. Disponível em: <http://link.estadao.com.br/noticias/cultura-digital,bancos-brasileiros-usarao-dadosreais-em-testes-de-blockchain-em-2018,70002128287>. Acesso em: 23 dez. 2017.

LOUREIRO, R. Blockchain, tecnologia da Bitcoin, seduz os bancos. Disponível em: $<$ https:// www.istoedinheiro.com.br/blockchain-tecnologia-da-bitcoin-seduz-os-bancos/>. Acesso em: 23 dez. 2017.

MARKOWITZ, H. Portfolio selection. The Journal of Finance, v. 7, n. 60, p. 77-91, 1952.

MORAES, M. Possível governo Temer dá esperanças de aquecimento da economia, dizem analistas. Disponível em: <http://economia.ig.com.br/2016-04-27/possivel-governo-temerda-esperancas-de-aquecimento-da-economia-dizem-analistas.html>. Acesso em: 7 jul. 2018.

NAKAMOTO, S. Bitcoin: A Peer-to-Peer Electronic Cash System. p. 1-9, 2008. Disponível em: <https://bitcoin.org/bitcoin.pdf>.

PFIFFELMANN, M.; ROGER, T.; BOURACHNIKOVA, O. When Behavioral Portfolio Theory meets Markowitz theory. Economic Modelling, v. 53, p. 419-435, 2016.

PRYBILA, C.; SCHULTE, S.; HOCHREINER, C.; WEBER, I. Runtime verification for business processes utilizing the Bitcoin blockchain. Future Generation Computer Systems, 2017.

RADU, R. I.; ISAI, V.; MIHAI, O. I. Considerations Regarding the Possibility of Emphasising the Virtual Currency Transactions in Accounting. In: Annals of “Dunarea de Jos" University of Galati Fascicle I. Economics and Applied Informatics Years, Anais...2017. 
SIMSER, J. Bitcoin and modern alchemy: in code we trust. Journal of Financial Crime, v. 22, n. 2, p. 156-169, 2015.

SYMITSI, E.; CHALVATZIS, K. J. The Economic Value of Bitcoin : A Portfolio Analysis of Currencies, Gold, Oil and Stocks. SSRN Electronic Journal, p. 1-46, 2018. Disponível em: <http://dx.doi.org/10.2139/ssrn.3127534>. Acesso em: 9 jul. 2018.

TAN, B.S.; LOW, K. Y. Bitcoin - Its Economics for Financial Reporting. Australian Accounting Review, v. 27, n. 2, p. 220-227, 2017.

TESOURO NACIONAL. Conheça o Tesouro Direto. Disponível em: <http://www.tesouro. gov.br/web/stn/tesouro-direto-conheca-o-tesouro-direto>. Acesso em: 20 abr. 2018a.

TESOURO NACIONAL. Cálculo da Rentabilidade dos Títulos Públicos ofertados no Tesouro Direto - LFT. Disponível em: <http://www.tesouro.fazenda.gov.br/ documents/10180/258262/Cálculo+da+Rentabilidade+dos+Títulos+Públicos+ofertados+via +Tesouro+Direto+-+LFT.pdf/582e2ca6-adab-459b-b841-ef33d4863b3b>. Acesso em: 20 abr. 2018b.

ULRICH, F. BITCOIN - A moeda na era digital. São Paulo: Instituto Ludwig von Mises Brasil, 2014.

ULRICH, F. Por que o bitcoin superou o ouro no Brasil. Disponível em: <http://www. infomoney.com.br/blogs/cambio/moeda-na-era-digital/post/5463393/por-que-bitcoinsuperou-ouro-brasil>. Acesso em: 27 out. 2017.

URQUHART, A. What Causes the Attention of Bitcoin? Economics Letters, v. 166, p. 40-44, 2018.

WU, C. Y.; PANDEY, V. K. The Value of Bitcoin in Enhancing the Efficiency of an Investor's Portfolio. Journal of Financial Planning, v. 27, n. 9, p. 44-52, 2014.

YEOH, P. Regulatory issues in blockchain technology. Journal of Financial Regulation and Compliance, v. 25, n. 2, p. 196-208, 2017. 